\title{
Protein Sequence Abnormality
}

National Cancer Institute

\section{Source}

National Cancer Institute. Protein Sequence Abnormality. NCI Thesaurus. Code C45594.

A change in the amino acid sequence of a wild-type protein. This alteration typically occurs due to a mutation of the gene that encodes the protein. The change in amino acid sequence may enhance, reduce, abolish or have no effect on protein activity. 